Onkologie 1996;19:1

\title{
Imprint, Vol. 19, No. 1, 1996
}

\section{Schriftleiturtg}

W. Queißer, Mannheim H. Huber, Wien

Fachschriftleitung

W. E. Berdel, Berlin E. Dühmke, München R. Hartenstein, München M. Kaufmann,

Frankfurt/M. H. Rübben, Essen P. Schlag, Berlin

Wissenschaftlicher Beirat

L. Bergmann, Frankfurt/M.

C. Bokemeyer, Tubingen

U. Creutzig, Münster

V. Diehl, Köln

P. Drings, Heidelberg

L. Edler, Heidelberg

G. Eisenbrand, Kaiserslautern

H. H. Fiebig, Freiburg

H. Gadner, Wien

K. Havemann, Marburg

F. Herrmann, Ulm

R. Herrmann, Basel

D. Hoelzer, Frankfurt/M.

H. J. Illiger, Oldenburg

R. Jakesz, Wien

U. R. Kleeberg, Hamburg R. Kreienberg, Ulm M. Lehnert, St. Gallen

H. Löffler, Kiel H. Ludwig, Wien H. J. Meyer, Hannover F.A. Muthny, Münster G. A. Nagel, Freiburg R. Parwaresch, Kiel

A. Pfleiderer, Freiburg

F. Porzsolt, Ulm

K. Possinger, Berlin H. Sauer, München

G. Schackert, Dresden

H.-J. Schmoll, Halle

R. Schulte-Hermann, Wien S. Seeber, Essen

B. Thürlimann, St. Gallen

W. Tilgen, Heidelberg

M. Wannenmacher, Heidelberg H. J. Weh, Hamburg H. Wilke, Essen M. Wirth, Dresden

Die Zeitschrift erscheint zweimonatlich; pro Jahr erscheint 1 Band zu je 6 Heften.

Bezugspreis für Jahrgang 19, 1996, DM 182,- I SFr 142,-, einschließlich MwSt., zu-züglich Poslgebühren. Der Abonnementpreis ist im voraus zahlbar. Das Abonne-ment der Zeitschrift läuft weiter, wenn es nicht spätestens 4 Wochen vor Abschluß eines Bandes abbestellt wird. 
Abonnementbestellungen können bei jeder Buchhandlung oder direkt beim Ver-lag aufgegebe $\pi$ werden:

Bundesrepublik Deutschland: S. Karger GmbH, Lörracher Sir. 16a, D-79115 Freiburg, Telefon (0761) 452070, Telefax (0761) 45207 14, Postgiro München 40080-807

Übrige Lander: S. Karger AG, Allschwilerstr. 10, Postfach, CH-4009 Basel, Telefon (061)30611

11, Telefax (061)3061234, E-Mail Kargerfe > Karger.ch.

Anzeigen

S. Karger Verlag für Mcdizín und Naturwissenschaften $\mathrm{GmbH}$,

Lörracher Str. 16 a, D-79115 Freiburg, Telefon (07 61) 452070.

Gültig ist die Preislistc Nr. 9 vom 1. Januar 1996.

Für den Inhalt außerhalb des redaktionellen Teiles (insbesondere Anzeigen, In-

dustrieinformationen, Pressezitate und Kongreßinformationen) übernchmen

Schriftleitung, Beirat und Verlag keine Gewähr.

Eine Markenbezeichnung kann warenzeichenrechtlich geschützt sein, auch wenn

bei ihrer Verwendung in dieser Zeitschrift das Zeichen ${ }^{\circledR}$ oder ein anderer Hinweis

auf etwa bestehende Schutzrechte fehlen sollte. Für Satzfehler, insbesondere bei

Dosierungsangaben, wird keine Gewähr übernommen.

Die Zeitschrift sowie alle in ihr cnthaltenen einzelnen Beiträge und Abbildungen sind urheberrechtlich geschützt. Jede Verwertung, die nicht ausdrücklich vom Ur-heberrechtsgesetz zugelassen ist, bedarf der vorherigen Zustimmung des Verlags. Das gilt insbesondere für Vervielfältigungen, Bearbeitungen, Übersetzungen, Mi-kroverfillmungen und die Einspeicherung und Verarbeitung in elektronischen Sy-stemen. Fotokopien dürfcn nur für den persönlichen Gebrauch als Einzelkopien hergestellt werden. Jede im Bereich eines gewerblichen

Unternehmens zulässig hergestellte oder benutzte Kopie dient gewerblichen Zwecken gem. § 54(2) UrhG und verpílichtet zur Gebührenzahlung an die Verwertungsgesellschaft WORT, Abt. VG Wissenschaft, Goethestraße 49, D-80336 München 2.

(C) Copyright 1996 by S. Karger

Verlag für Medizin und Naturwissenschaften GmbH.

Lörracher Str. 16 a

D-79115 Freiburg

Verlagsleitung und presserechtlich verantwortlich: Sibylle Hopf Assislenz: Susanne Mcister Herstcllung: Georg Brunner Anzeigenverwaltung: Christiane Opitz

Satz und Druck: Walter Biering GmbH Grafischer Betrieb Freisinger Landstraße 21 D-80939 München

KARGER 